\title{
(3) Niraparib Maintenance Treatment Improves Time Without Symptoms or Toxicity (TWiST) Versus Routine Surveillance in Recurrent Ovarian Cancer: A TWiST Analysis of the ENGOT-OV16/ NOVA Trial
}

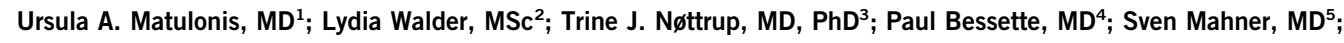
Marta Gil-Martin, MD $^{6}$; Elsa Kalbacher, MD $^{7}$; Jonathan A. Ledermann, MD $^{8}$; Robert M. Wenham, MD ${ }^{9}$; Kathrine Woie, MD, PhD ${ }^{10}$; Susie Lau, MD ${ }^{11}$; Frederik Marmé, MD, PhD ${ }^{12}$; Antonio Casado Herraez, MD, $\mathrm{PhD}^{13}$; Anne-Claire Hardy-Bessard, $\mathrm{MD}^{14}$; Susana Banerjee, PhD, MA ${ }^{15}$; Gabriel Lindahl, MD ${ }^{16}$; Benedict Benigno, MD ${ }^{17}$; Joseph Buscema, MD ${ }^{18}$; Karin Travers, DSc ${ }^{19}$; Holly Guy, $\mathrm{MSc}^{2}$; and Mansoor R. Mirza, $\mathrm{MD}^{3}$

ASSOCIATED CONTENT

Data Supplement

Author affiliations and support information (if applicable) appear at the end of this article.

Accepted on July 23 , 2019 and published at jco.org on

September 16, 2019: DOI https://doi.org/10. $1200 / J C 0.19 .00917$

Clinical trial information: NCT01847274.

PURPOSE This study estimated time without symptoms or toxicity (TWiST) with niraparib compared with routine surveillance (RS) in the maintenance treatment of patients with recurrent ovarian cancer.

PATIENTS AND METHODS Mean progression-free survival (PFS) was estimated for niraparib and RS by fitting parametric survival distributions to Kaplan-Meier data for 553 patients with recurrent ovarian cancer who were enrolled in the phase III ENGOT-OV16/NOVA trial. Patients were categorized according to the presence or absence of a germline BRCA mutation - gBRCAmut and non-gBRCAmut cohorts. Mean time with toxicity was estimated based on the area under the Kaplan-Meier curve for symptomatic grade 2 or greater fatigue, nausea, and vomiting adverse events (AEs). Time with toxicity was the number of days a patient experienced an AE post-random assignment and before disease progression. TWiST was estimated as the difference between mean PFS and time with toxicity. Uncertainty was explored using alternative PFS estimates and considering all symptomatic grade 2 or greater AEs.

RESULTS In the gBRCAmut and non-gBRCAmut cohorts, niraparib treatment resulted in a mean PFS benefit of 3.23 years and 1.44 years, respectively, and a mean time with toxicity of 0.28 years and 0.10 years, respectively, compared with RS. Hence, niraparib treatment resulted in a mean TWiST benefit of 2.95 years and 1.34 years, respectively, compared with RS, which is equivalent to more than four-fold and two-fold increases in mean TWiST between niraparib and RS in the gBRCAmut and non-gBRCAmut cohorts, respectively. This TWiST benefit was consistent across all sensitivity analyses, including modeling PFS over 5-, 10-, and 15-year time horizons.

CONCLUSION Patients who were treated with niraparib compared with RS experienced increased mean TWiST. Thus, patients who were treated with niraparib in the ENGOT-OV16/NOVA trial experienced more time without symptoms or symptomatic toxicities compared with control.

J Clin Oncol 37:3183-3191. @ 2019 by American Society of Clinical Oncology

Creative Commons Attribution Non-Commercial No Derivatives 4.0 License (c) (i) $(-)$

\section{INTRODUCTION}

Ovarian cancer (OC) was diagnosed in 240,000 women worldwide in 2012. ${ }^{1}$ Most women are diagnosed with advanced cancer and prognosis is poor, which is reflected by OC being one of the most deadly forms of women's cancer, with a 5-year survival rate of $47.4 \%$ and approximately 151,917 deaths in $2012 .^{1,2}$ The standard of care for first-line treatment is platinumand taxane-based chemotherapy, which results in high initial response rates; however, relapse rates for women who are diagnosed with advanced OC can be up to $95 \% .^{3,4}$

Given the lack of a cure for recurrent $\mathrm{OC}(\mathrm{ROC})$ and the negative impact on patient quality of life (QoL), there is a need for effective maintenance treatments that delay disease progression without the toxic effects that offset this benefit and affect patient QoL. The poly(ADP-ribose) polymerase inhibitor (PARPi) niraparib is approved by the US Food and Drug Administration and European Medicines Agency for use as maintenance treatment in 
patients with recurrent epithelial ovarian, fallopian tube, or primary peritoneal cancer who have achieved complete or partial response to platinum-based chemotherapy. 5,6 In the randomized controlled phase III ENGOT-OV16/NOVA trial of niraparib, patients were categorized according to the presence or absence of a germline BRCA mutation-gBRCAmut and non-gBRCAmut cohorts. Results demonstrated that niraparib treatment significantly extended median progressionfree survival (PFS) in the gBRCAmut (21.0 months $v 5.5$ months; $P<.001$ ) and non-gBRCAmut cohorts (9.3 months $V$ 3.9 months; $P<.001)^{7}$

The ENGOT-OV16/NOVA trial demonstrated that patient QoL remained stable during treatment and the preprogression period, with no significant differences observed between treatment arms. ${ }^{8}$ Therefore, niraparib treatment prolongs PFS compared with placebo and maintains QoL. To explore this benefit further, this analysis estimated the time without symptoms or toxicity (TWiST) between niraparib and routine surveillance (RS) in patients with gBRCAmut and non-gBRCAmut tumors who were enrolled in ENGOT-OV16/NOVA. TWiST is an established methodology that partitions PFS into two health states and evaluates the tradeoff between treatment toxicity and time to progression $^{9,10}$; therefore, TWiST provides an estimate of time when a patient is free from progression and toxicity and thus likely to maintain a good QoL.

\section{PATIENTS AND METHODS}

\section{Data Source}

This analysis used data from the gBRCAmut and nongBRCAmut cohorts of ENGOT-OV16/NOVA (ClinicalTrials.gov identifier: NCT01847274). Eligible patients were age 18 years or older; had histologically diagnosed ovarian, fallopian tube, or primary peritoneal cancer with predominantly high-grade serous histologic features; and received two or more platinum-based chemotherapy regimens. Patients must have had platinum-sensitive disease-complete or partial response and disease progression-more than 6 months after their penultimate platinum-based chemotherapy. Patients were randomly assigned 2:1 to receive once-daily niraparib $300 \mathrm{mg}$ or placebo. ${ }^{7}$ Comparison with placebo was chosen to reflect the impact of niraparib on clinical practice. As such, placebo is referred to as RS from here on. The ENGOT-OV16/ NOVA primary end point was PFS, which was defined as the time from treatment randomization to the earliest date of disease progression or death from any cause. Secondary end points included patient-reported outcomes, chemotherapy-free interval, PFS on the next line of therapy, time to first and second subsequent therapy, and overall survival. Efficacy data were analyzed in the intention-totreat population, which was defined as all patients who underwent random assignment in each cohort. Safety data were analyzed in the safety population, defined as all patients who received one or more doses of treatment. ${ }^{7}$

\section{Study Participants}

This analysis was performed on the intention-to-treat population of the gBRCAmut (niraparib, $\mathrm{n}=138$; $\mathrm{RS}, \mathrm{n}=$ 65) and non-gBRCAmut cohorts (niraparib, $n=234$; RS, $\mathrm{n}=116$ ) from ENGOT-OV16/NOVA. Patient baseline characteristics were balanced across cohorts (Data Supplement Table S1). ${ }^{7}$ One patient in the gBRCAmut cohort was excluded from the niraparib data set as the patient had only received first-line chemotherapy before initiating niraparib. One patient from the non-gBRCAmut cohort was excluded from the RS data set as no prior therapy line was recorded.

\section{Statistical Analysis}

Statistical analysis was performed using RStudio, version 1.138 (Boston, MA). ${ }^{11}$

PFS. Patient-level data (database lock: June 20, 2016) from ENGOT-OV16/NOVA were used to derive KaplanMeier PFS curves for niraparib and RS for the gBRCAmut and non-gBRCAmut cohorts.

The time horizon for PFS extrapolation was based on expert clinical opinion such that patients could be progression free for up to 20 years. In addition, a recent National Institute for Health and Care Excellence (NICE) appraisal reported that it is biologically plausible for patients receiving niraparib to be progression free more than 10 years. ${ }^{12}$ Therefore, the base case analysis assumed that patients could be progression free for up to 20 years. In addition, the assumption that patients could be progression free for up to 5, 10, and 15 years was explored in sensitivity analyses

PFS extrapolation was performed over a 20-year time horizon by fitting standard parametric survival distributions to niraparib and RS Kaplan-Meier PFS data. NICE Decision Support Unit guidelines were followed. ${ }^{13}$ The best-fitting distribution between treatment arms for each cohort, referred to as the base case, was selected on the basis of clinical plausibility and statistical and visual fit. The determination of the best-fitting distribution is further reported in the Data Supplement.

The generalized gamma distribution was identified as the statistically best-fitting distribution across treatment arms for both cohorts. Visual inspection confirmed that the generalized gamma distribution fit the data well (Fig 1). Clinical plausibility was assessed by comparing 5-year survival estimates with long-term survival available in platinum-sensitive ROC (PSROC) for PARPi. Study 19 olaparib data demonstrated that approximately $16 \%$ and $14 \%$ of patients with BRCAmut and BRCA wild-type tumors, respectively, remained on treatment-that is, progression free-at 5 years. ${ }^{14}$ Of the six standard parametric survival distributions, the generalized gamma distribution most closely modeled long-term survival from Study 19, with $22 \%$ and $13 \%$ of patients progression free at 5 years in the $g B R C A m u t$ and non-gBRCAmut cohorts, respectively. 


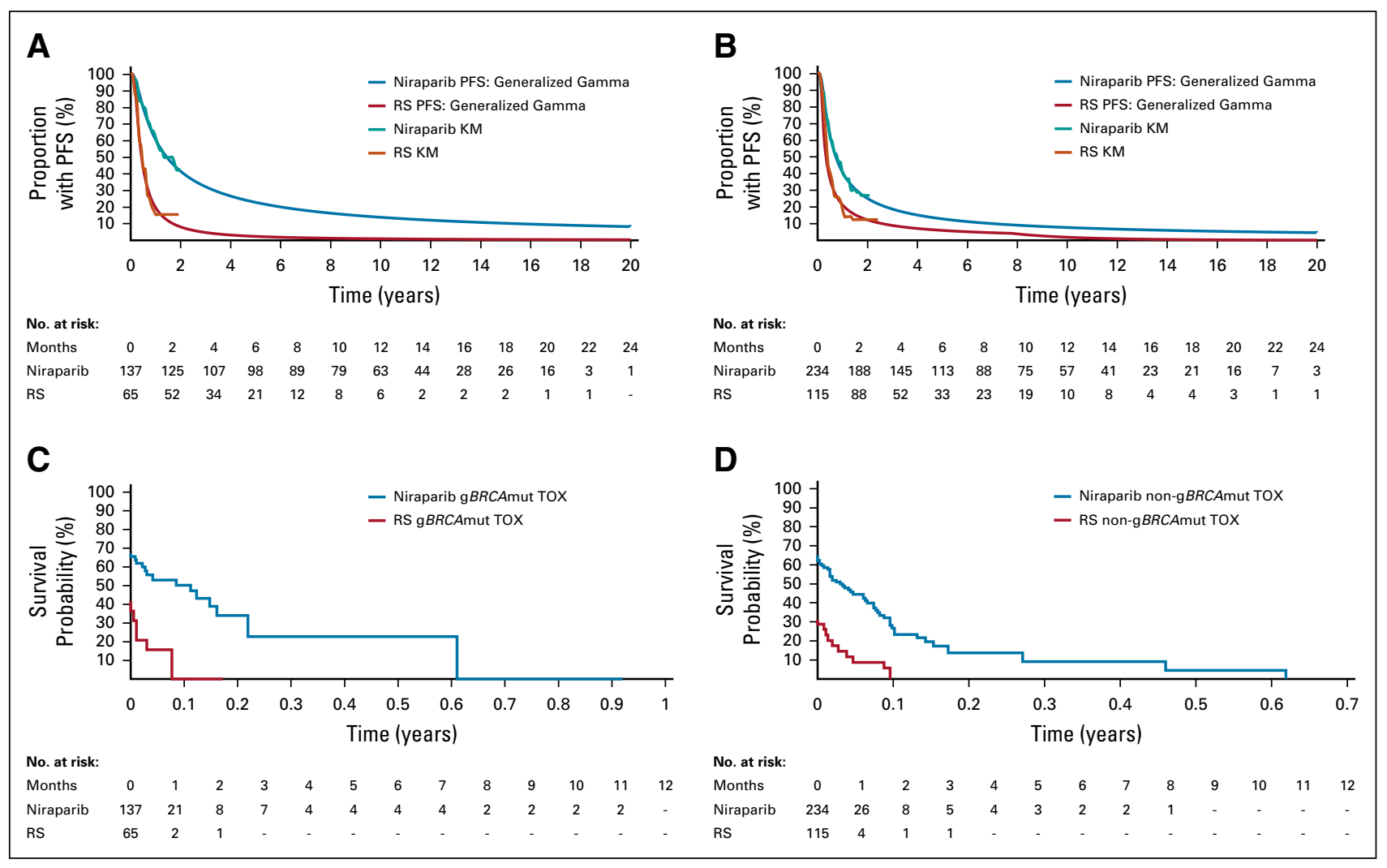

FIG 1. Kaplan-Meier (KM) curves for progression-free survival (PFS) and generalized gamma distributions over a 20-year time horizon for the (A) gBRCAmut and (B) non-gBRCAmut cohorts, and KM curves for time with toxicity (TOX) for the (C) gBRCAmut and (D) non-gBRCAmut cohorts. gBRCAmut, germline $B R C A$ mutation; RS, routine surveillance.

Thus, this analysis used the generalized gamma distribution for the base case PFS extrapolation.

Mean PFS for niraparib and RS was calculated as the area under the curve (Data Supplement, Equation S1).

Toxicity and TWiST. In ENGOT-OV16/NOVA, safety was assessed by adverse event (AE) monitoring, laboratory tests, vital signs, and physical examinations. ${ }^{7}$ AEs were collected from the time of consent through treatment discontinuation as a result of disease progression or unacceptable toxicity. New serious AEs were collected for 30 days after treatment discontinuation. ${ }^{7}$ Few patients discontinued therapy because of AEs (niraparib, 15\%; placebo, $2 \%$ ), and the most common AEs were managed with dose reductions and treatment interruptions. ${ }^{7}$ Disease progression was the primary reason for treatment discontinuation (niraparib, 52\%; placebo, 81\%).

To estimate the mean time with toxicity, three symptomatic grade 2 or greater AEs which patients experienced in ENGOT-OV16/NOVA were included: fatigue (niraparib, $\mathrm{n}=$ 80 [21.8\%]; RS, $\mathrm{n}=15$ [8.4\%]; $P<.001$ ), nausea (niraparib, $\mathrm{n}=78$ [21.3\%]; RS, $\mathrm{n}=15$ [8.4\%]; $P=.003)$, and vomiting (niraparib, $\mathrm{n}=25$ [6.8\%]; RS, $\mathrm{n}=8$ [4.5\%]; $P=$ .3413). These AEs were consistent with those selected in the published SOLO-2 trial TWiST analysis ${ }^{10}$ and were among some of the most common symptomatic AEs experienced by patients in ENGOT-OV16/NOVA. Therefore, it could be expected that these AEs would have the greatest impact on QoL. The impact of hematologic AEs-for example, anemia, neutropenia, and thrombocytopenia-was not assessed, as a previous analysis concluded that they had no significant effect on QoL in ENGOT-OV16/NOVA. ${ }^{8}$

Time with toxicity for each patient was calculated as the number of days a patient experienced grade 2 or greater fatigue, nausea, or vomiting after random assignment and before disease progression. The number of days a patient experienced one of these AEs was calculated by subtracting the $A E$ end date from the start date (Eq 1 ).

$$
A E \text { duration }=\text { end date }- \text { start date. }
$$

Multiple occurrences of these AEs experienced by a patient over the same time period were not counted twice. As such, the earliest start date and the latest end date were taken. In cases in which only the month and year were reported for the AE start or end date, data were imputed as the first day of that month (niraparib, $n=1$; RS, $n=1$ ). AEs were excluded if they ended before randomization or began after disease progression. For AEs that began before randomization, the start date was set to the patient's random assignment date. For AEs that extended beyond disease progression, the end date was truncated at disease 
progression. Time with toxicity was calculated for 202 patients (niraparib, $n=137$; RS, $n=65$ ) and 349 patients (niraparib, $\mathrm{n}=234$; RS, $\mathrm{n}=115$ ) in the gBRCAmut and non-gBRCAmut cohorts, respectively.

Kaplan-Meier time with toxicity curves were estimated for niraparib and RS for both cohorts (Fig 1). Mean time with toxicity was calculated as the restricted Kaplan-Meier mean (Data Supplement, Equation S1).

Mean TWiST was estimated as the difference between mean PFS and mean time with toxicity for each arm (Eq 2). The mean TWiST difference between niraparib and RS was calculated as the difference between mean TWiST for niraparib and RS.

Mean TWiST $=$ mean PFS - mean time with toxicity.

\section{Sensitivity Analyses}

Sensitivity analyses assessed the impact of alternative PFS distributions, PFS time horizon, and the inclusion of all symptomatic grade 2 or greater AEs on the mean TWiST difference between niraparib and RS.

To assess the impact of different PFS distributions, alternative distributions were selected by considering statistical and visual fit and clinical plausibility. Two additional standard parametric survival distributions-log-normal and log-logistic-were explored as they closely modeled 5-year survival data available from Study 19 (log-normal: gBRCAmut, 11.46\%; non-gBRCAmut, 2.91\%; log-logistic: gBRCAmut, 11.03\%; non-gBRCAmut, 3.98\%). ${ }^{14}$ In addition, both distributions were a good statistical and visual fit to the observed data for both cohorts (Data Supplement, Tables S2-S3, Fig. S1-S4).

Flexible PFS distributions were also explored per NICE Decision Support Unit guidelines and published literature. ${ }^{13,15}$ Two distributions-normal $k=1$ and odds $k=3$-were explored as they closely modeled 5-year survival data available from Study 19 for both cohorts (normal $\mathrm{k}=1$ : gBRCAmut, $17.75 \%$; non-gBRCAmut, 9.38\%; odds $\mathrm{k}=3$ : gBRCAmut, $20.54 \%$, non-gBRCAmut, 9.21\%). ${ }^{14}$ Both distributions were a good statistical fit to the observed data for both cohorts (Data Supplement Tables S4-S5). The Data Supplement (Fig S5-S8) shows that distributions fit the observed data well.

In addition, sensitivity analyses were performed on the PFS time horizon, where all patients were assumed to have experienced disease progression by 5, 10, or 15 years.

Finally, the inclusion of all symptomatic grade 2 or greater AEs experienced by more than $10 \%$ of patients in either treatment arm of ENGOT-OV16/NOVA (Table 1) was explored.

\section{Validation}

The TWiST methodology underwent internal and external validation. PFS extrapolation estimates were validated against published long-term survival data in PSROC for PARPi. ${ }^{14}$ In addition, modeled outcomes were validated using median survival time to align with the reporting of ENGOT-OV16/NOVA, which is standard practice in clinical trials. The analysis was conducted internally by two independent analysts (L.W. and H.G.), and the inputs were checked for accuracy by TESARO: A GSK Company (Waltham, MA). An external statistician reviewed the approach and provided suggestions for improvement, which included Kaplan-Meier toxicity curves and flexible PFS methodology. All feedback obtained was incorporated into the final analysis and this publication.

\section{RESULTS}

\section{Base Case Results}

Base case PFS extrapolation distributions-generalized gamma over 20 years - and Kaplan-Meier time with toxicity curves are presented in Figure 2 for niraparib and RS. Table 2 lists mean PFS, mean time with toxicity, and the estimated mean TWiST difference between niraparib and RS for both cohorts.

For the gBRCAmut cohort, niraparib was associated with a mean PFS benefit of 4.14 years and a mean time with toxicity of 0.31 years, which is equivalent to a mean TWiST of 3.83 years. In addition, RS was associated with mean PFS and mean time with toxicity of 0.91 and 0.03 years, respectively, which is equivalent to a mean TWiST of 0.88 years. Hence, niraparib treatment resulted in a mean TWiST benefit of 2.95 years compared with RS.

For the non-gBRCAmut cohort, niraparib was associated with a mean PFS of 2.59 years and a mean time with toxicity of 0.13 years, which is equivalent to a mean TWiST of 2.46 years. In addition, RS was associated with mean PFS and mean time with toxicity of 1.14 and 0.02 years, respectively, which is equivalent to a mean TWiST of 1.12 years. Hence, niraparib treatment resulted in a mean TWiST benefit of 1.34 years compared with RS.

\section{Sensitivity Analyses}

For all sensitivity analyses, niraparib demonstrated a consistent mean TWiST benefit compared with RS when examining PFS at 5, 10, and 15 years (Table 2 and Data Supplement Fig S9-S25). When extrapolated PFS was modeled with more conservative estimates, mean TWiST benefit ranged from 1.15 to 3.65 years and 0.52 to 1.23 years for the gBRCAmut and non-gBRCAmut cohorts, respectively. When all symptomatic grade 2 or greater $A E s$ were included, mean TWiST benefit was 3.01 and 1.22 for the gBRCAmut and non-gBRCAmut cohorts, respectively.

\section{Validation}

Median modeled and observed PFS from ENGOT-OV16/ NOVA were similar for both niraparib and RS for gBRCAmut (niraparib: modeled $v$ observed, $21.0 v 21.0$ months; RS: modeled vobserved, $5.52 v 5.5$ months) and non-gBRCAmut 
TABLE 1. Incidence of All Symptomatic Grade $\geq 2$ Adverse Events Experienced by $\geq 10 \%$ of Patients in Either Treatment Arm by Cohort of ENGOT-OV16/NOVA

gBRCAmut Cohort

Non-gBRCAmut Cohort

\begin{tabular}{|c|c|c|c|c|c|c|}
\hline \multirow[b]{2}{*}{ Adverse Event } & & \\
\hline & Niraparib $(n=136)$ & RS $(n=65)$ & $P$ & Niraparib $(n=231)$ & RS $(n=114)$ & $P$ \\
\hline Fatigue & $43(31.6)$ & $8(12.3)$ & .0031 & 73 (31.6) & $13(11.4)$ & $<.001$ \\
\hline Nausea & $30(22.1)$ & $6(9.2)$ & .0303 & $48(20.8)$ & $9(7.9)$ & .0020 \\
\hline Hypertension & $26(19.1)$ & $4(6.2)$ & .0189 & $38(16.5)$ & $3(2.6)$ & $<.001$ \\
\hline Constipation & $17(12.5)$ & $2(3.1)$ & .0384 & $26(11.3)$ & $6(5.3)$ & .0780 \\
\hline Headache & 15 (11.0) & $2(3.1)$ & .0628 & $4(1.7)$ & $1(0.9)$ & 1.0000 \\
\hline Vomiting & $14(10.3)$ & $1(1.5)$ & .0405 & $11(4.8)$ & $7(6.1)$ & .6116 \\
\hline Abdominal pain & $10(7.4)$ & $5(7.7)$ & 1.0000 & $21(9.1)$ & $12(10.5)$ & .6990 \\
\hline Urinary tract infection & $8(5.9)$ & $4(6.2)$ & 1.0000 & $16(6.9)$ & $4(3.5)$ & .2313 \\
\hline Dyspepsia & $7(5.1)$ & $1(1.5)$ & .4412 & $5(2.2)$ & $1(0.9)$ & .6679 \\
\hline Back pain & $7(5.1)$ & $2(3.1)$ & .7210 & $10(4.3)$ & $5(4.4)$ & 1.0000 \\
\hline Dyspnea & $6(4.4)$ & 0 & .1799 & $16(6.9)$ & $3(2.6)$ & .1327 \\
\hline Decreased appetite & $6(4.4)$ & $1(1.5)$ & .4319 & $16(6.9)$ & $2(1.8)$ & .0425 \\
\hline Diarrhea & $5(3.7)$ & $2(3.1)$ & 1.0000 & $8(3.5)$ & $5(4.4)$ & .7653 \\
\hline Arthralgia & $5(3.7)$ & $2(3.1)$ & 1.0000 & $8(3.5)$ & $3(2.6)$ & 1.0000 \\
\hline Insomnia & $5(3.7)$ & 0 & .1770 & $13(5.6)$ & $1(0.9)$ & .0411 \\
\hline Nasopharyngitis & $4(2.9)$ & $1(1.5)$ & 1.0000 & $5(2.2)$ & $1(0.9)$ & .6679 \\
\hline Abdominal distension & $2(1.5)$ & $1(1.5)$ & 1.0000 & $6(2.6)$ & $4(3.5)$ & .7353 \\
\hline Dizziness & $2(1.5)$ & $1(1.5)$ & 1.0000 & $6(2.6)$ & 0 & .1836 \\
\hline Cough & $2(1.5)$ & 0 & 1.0000 & $3(1.3)$ & 0 & .5536 \\
\hline Myalgia & $1(0.7)$ & $1(1.5)$ & .5433 & $5(2.2)$ & $2(1.8)$ & 1.0000 \\
\hline Palpitations & $1(0.7)$ & 0 & 1.0000 & $5(2.2)$ & 0 & .1753 \\
\hline
\end{tabular}

NOTE. Data provided as No. (\%) unless otherwise noted.

Abbreviations: gBRCAmut, germline $B R C A$ mutation; RS, routine surveillance.

cohorts (niraparib: modeled $v$ observed, 9.26 v 9.3 months; RS: modeled $v$ observed, $3.91 v 3.9$ months). ${ }^{7}$

\section{DISCUSSION}

This analysis sought to evaluate the difference in TWiST between niraparib and RS in the maintenance treatment of patients with PSROC. TWiST is the amount of timemeasured in years - during which patients are progression and toxicity free and thus likely to maintain good QoL. Mean PFS extrapolated from ENGOT-OV16/NOVA over a 20-year time horizon demonstrated that patients receiving niraparib, compared with $\mathrm{RS}$, experienced increased mean PFS in both cohorts. Sensitivity analyses were performed at additional clinically relevant 5-, 10-, and 15-year PFS timeframes, demonstrating improved TWIST in niraparibtreated patients. Furthermore, subtracting the mean time with toxicity for grade 2 or greater fatigue, nausea, and vomiting from mean PFS resulted in an increased mean TWiST. Base case results showed that niraparib was associated with a mean TWiST benefit compared with RS. These TWiST benefits demonstrate that patients receiving niraparib will maintain good QoL. Specifically, compared with RS, patients with gBRCAmut and non-gBRCAmut tumors who receive niraparib will gain 2.95 years and 1.34 years, respectively, during which they are progression and toxicity free. In addition, results demonstrate that the mean TWiST for niraparib-treated patients is more than four-fold and two-fold greater than the mean TWiST for patients receiving $\mathrm{RS}$ in the gBRCAmut and non-gBRCAmut cohorts, respectively.

Robustness of the niraparib TWiST benefit compared with RS was confirmed through consistency across all sensitivity analyses in both cohorts. The greatest mean TWiST benefit observed in these sensitivity analyses for niraparib, compared with RS, was 3.65 years and 1.23 years for the gBRCAmut and non-gBRCAmut cohorts, respectively. When alternative PFS estimates were considered, niraparib mean TWiST was up to five-fold and two-fold greater than the RS mean TWiST in the gBRCAmut and non-gBRCAmut cohorts, respectively. In addition, when all grade 2 or greater symptomatic AEs were considered, niraparib mean TWiST was more than four-fold and two-fold greater than the RS mean TWiST in the gBRCAmut and non-gBRCAmut cohorts, respectively. As a result of this TWiST benefit, 


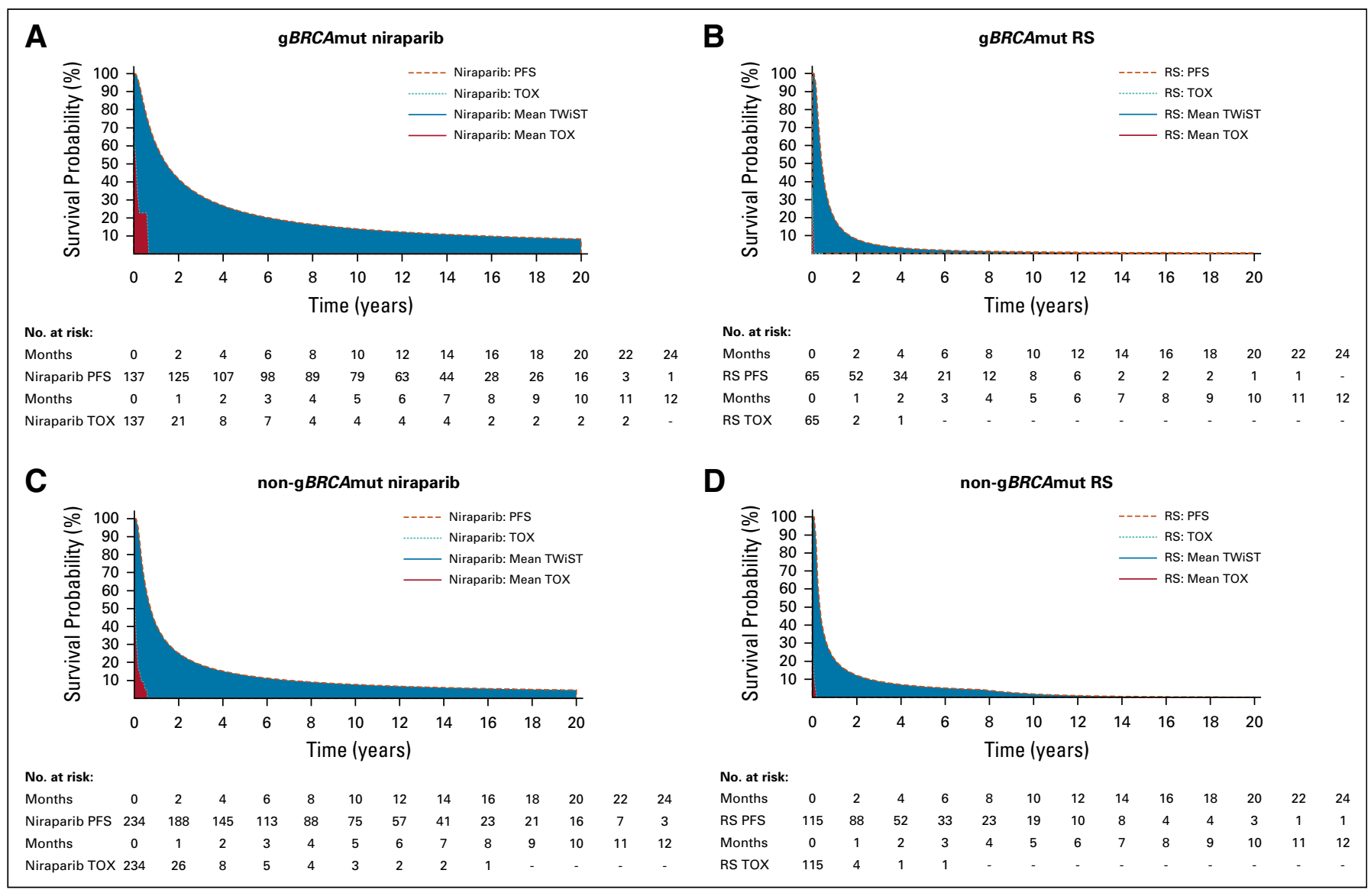

FIG 2. Kaplan-Meier curves for time with toxicity (TOX), mean progression-free survival (PFS) generalized gamma distributions, and mean time without symptoms or toxicity (TWiST) for (A) niraparib and (B) routine surveillance (RS) in the gBRCAmut cohort and for (C) niraparib and (D) RS in the nongBRCAmut cohort over a 20-year time horizon. gBRCAmut, germline BRCA mutation.

patients receiving niraparib spend more time progression and toxicity free with respect to grade 2 or greater fatigue, nausea, and vomiting than do patients receiving RS. This analysis demonstrates that niraparib treatment maintained QoL among progression-free patients, which is consistent with a previous analysis that showed that patients receiving niraparib had stable QoL during the preprogression period. Furthermore, all symptoms as measured by the Functional Assessment of Cancer Therapy-Ovarian Symptom Index, with the exception of nausea, remained stable or improved over time with niraparib treatment. ${ }^{8}$ ROC has been associated with a negative psychological and physical impact on patients, particularly as patients receive additional lines of subsequent chemotherapy. ${ }^{16}$ Therefore, as PFS decreases with each subsequent line of chemotherapy, ${ }^{17}$ and subsequent chemotherapy is associated with burdensome AEs and a negative impact on QoL, there is a need for an alternative to RS for the maintenance treatment of patients with PSROC. Effective PARPis, such as niraparib, which can extend PFS without negatively affecting QoL, can significantly improve the prognosis of PSROC compared with RS.
This publication reports one of the first TWiST analyses conducted for a PARPi for the maintenance treatment of patients with PSROC and is the first TWiST analysis that evaluates both gBRCAmut and non-gBRCAmut populations. The only other published TWiST analysis-by Friedlander et $\mathrm{al}^{10}$ - evaluated the TWiST difference between olaparib and placebo in the SOLO-2 trial; however, these results are not comparable with those presented here as a result of a different methodology used. Friedlander et $\mathrm{al}^{10}$ used PFS trial data only, whereas our study extrapolated PFS. Furthermore, the TWiST difference between olaparib and placebo was limited to gBRCAmut patients only because of trial eligibility. ${ }^{10}$

The main strength of this analysis is that the clinical evidence base can be considered to be representative of real-world patients with PSROC as data were sourced directly from patients with PSROC who were enrolled in ENGOT-OV16/NOVA. ${ }^{7}$ In addition, PFS distributions modeled clinically realistic estimates for PFS, which closely match published long-term survival data reported in PSROC for PARPi. ${ }^{14}$ Furthermore, this analysis considered the long-term TWiST difference through the 


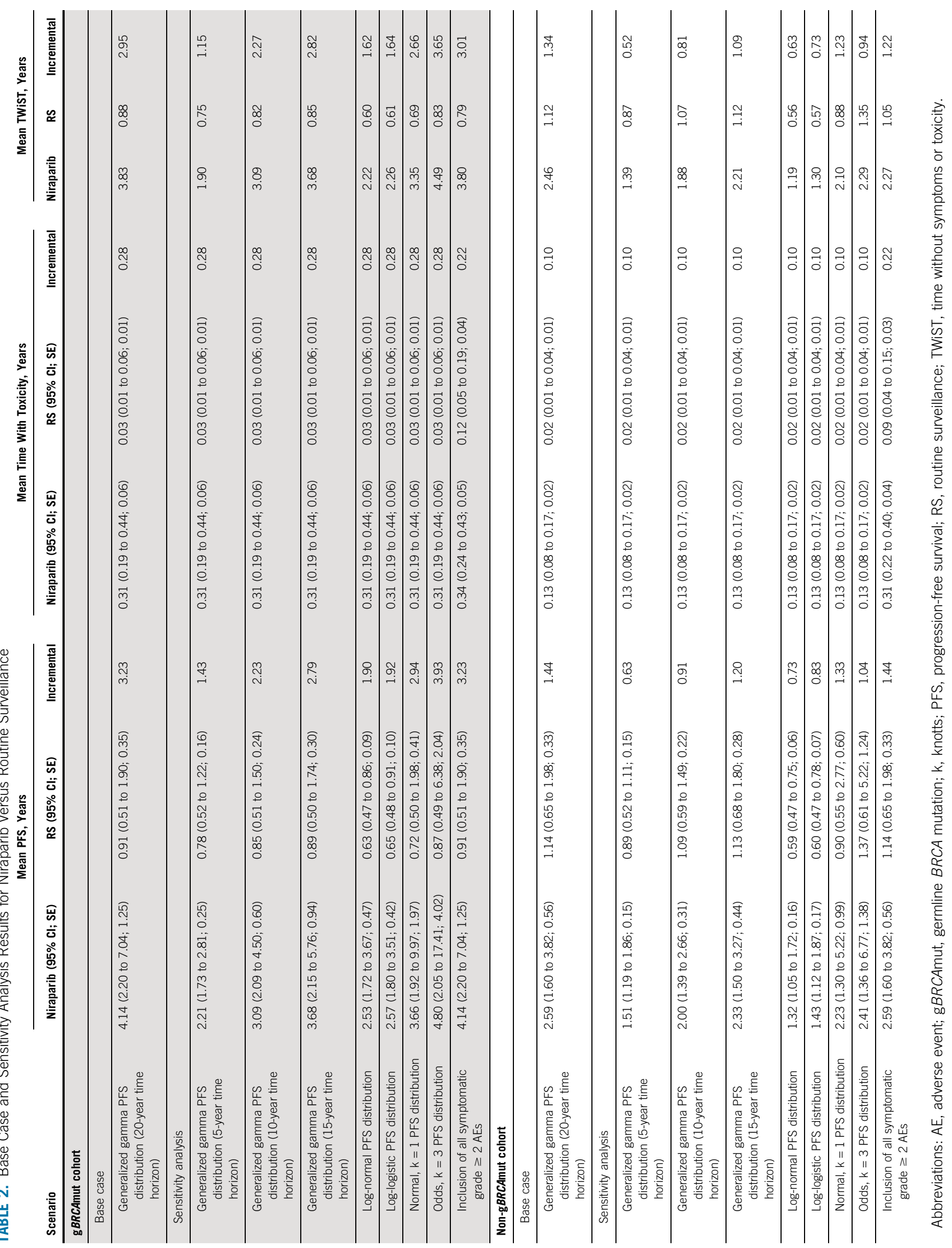


extrapolation of ENGOT-OV16/NOVA Kaplan-Meier PFS data, as opposed to comparing mean time with toxicity with restricted mean PFS only, thereby estimating the complete TWiST benefit expected for patients. Finally, this analysis has been demonstrated to be robust, as a TWiST benefit with niraparib was consistent across all sensitivity analyses performed.

This analysis has some limitations. The base case PFS was extrapolated over 20 years. Although it is unrealistic that long-term PFS would align with this cutoff point, this was based on expert clinical opinion and was accepted in two previous health technology appraisal submissions. ${ }^{18,19}$ Furthermore, the impact of alternative PFS estimates was explored through sensitivity analyses. Results indicated that patients who were treated with niraparib still experienced a TWiST benefit compared with RS even when more conservative PFS estimates were modeled. An additional limitation is that only the most common symptomatic non-hematologic grade 2 or greater AEs were included in the base case analysis. However, all symptomatic grade 2 or greater AEs experienced by more than $10 \%$ of ENGOT-OV16/NOVA patients was explored in a sensitivity analysis. These results demonstrated that niraparib-treated patients continue to experience an

\section{AFFILIATIONS}

${ }^{1}$ Dana-Farber Cancer Institute, Boston, MA

${ }^{2}$ FIECON, Ltd, St Albans, United Kingdom

${ }^{3}$ Nordic Society of Gynaecological Oncology and Copenhagen University Hospital, Copenhagen, Denmark

${ }^{4} \mathrm{PMHC}$ and University of Sherbrooke, Sherbrooke, Québec, Canada

${ }^{5}$ Arbeitsgemeinschaft Gynäkologische Onkologie and University of

Munich, Munich, Germany

${ }^{6}$ Grupo Español de Investigación en Cáncer de Ovario and Institut Català d'Oncologia-IDIBELL, L'Hospitalet, Barcelona, Spain

${ }^{7}$ Groupe d'Investigateurs Nationaux pour l'Etude des Cancers Ovariens and University Hospital Besançon, Besançon, France

${ }^{8}$ National Cancer Research Institute and University College London

Cancer Institute, London, United Kingdom

${ }^{9} \mathrm{H}$. Lee Moffitt Cancer Center, Tampa, FL

${ }^{10}$ Nordic Society of Gynaecological Oncology and Haukeland University Hospital, Bergen, Norway

${ }^{11} \mathrm{PMHC}$ and Jewish General Hospital, Montreal, Québec, Canada

${ }^{12}$ Arbeitsgemeinschaft Gynäkologische Onkologie and Universitätsklinikum Heidelberg, Heidelberg, Germany

${ }^{13}$ Grupo Español de Investigación en Cáncer de Ovario and Hospital Universitario San Carlos, Madrid, Spain

${ }^{14}$ Groupe d'Investigateurs Nationaux pour l'Etude des Cancers Ovariens and Centre Amoricain D'Oncologie, Paris, France

${ }^{15}$ National Cancer Research Institute and The Royal Marsden NHS

Foundation Trust and Institute of Cancer Research, London, United Kingdom

${ }^{16}$ Nordic Society of Gynaecological Oncology and Linköping University Hospital, Linköping, Sweden

${ }^{17}$ Northside Hospital, Atlanta, GA

${ }^{18}$ Arizona Oncology, Tucson, AZ

${ }^{19}$ TESARO: A GSK Company, Waltham, MA increased mean TWiST compared with RS, with the gBRCAmut mean TWiST increasing with respect to the base case analysis because of a smaller proportional increase in niraparib toxicity compared with RS. This is in agreement with results of a previous analysis in which a higher proportion of patients receiving RS reported pain compared with niraparib-treated patients. ${ }^{8}$

Future analyses could include a quality-adjusted TWiST analysis when mature overall survival data are available from ENGOT-OV16/NOVA. Moreover, an analysis of the safety profile of niraparib in ENGOT-OV16/NOVA recommended that patients with a baseline body weight of less than $77 \mathrm{~kg}$ or platelet count less than $150,000 / \mu \mathrm{L}$ may benefit from a 200-mg per day starting dose. ${ }^{20}$ Subsequent investigations could focus on the TWiST difference with this alternative starting dose.

TWiST estimates from ENGOT-OV16/NOVA indicate that niraparib-treated patients experienced increased mean TWiST compared with patients receiving RS. Therefore, patients receiving niraparib compared with control in ENGOTOV16/NOVA experienced more time without symptoms or symptomatic toxicities and thus have the potential to experience longer undiminished QoL.

\section{CORRESPONDING AUTHOR}

Ursula A. Matulonis, MD, Dana-Farber Cancer Institute, 450 Brookline Ave, Boston, MA 02115; Twitter: @DrMatulonis; e-mail:

ursula_matulonis@dfci.harvard.edu, and Holly Guy, FIECON Ltd, 3

College Yard, Lower Dagnall Street, St Albans, Hertfordshire, AL3 4PA, United Kingdom; Tel: +44 1727225 601; e-mail: holly.guy@fiecon.com.

\section{PRIOR PRESENTATION}

Presented at the 2019 Society of Gynecologic Oncology Annual Meeting, Honolulu, HI, March 16, 2019; and ISPOR Europe, Barcelona, Spain, November 14, 2018.

\section{SUPPORT}

Supported by TESARO: A GSK Company.

AUTHORS' DISCLOSURES OF POTENTIAL CONFLICTS OF INTEREST AND DATA AVAILABILITY STATEMENT

Disclosures provided by the authors and data availability statement (if applicable) are available with this article at DOI https://doi.org/10.1200/ JC0.19.00917

\section{AUTHOR CONTRIBUTIONS}

Conception and design: Ursula A. Matulonis, Lydia Walder, Jonathan A. Ledermann, Frederik Marmé, Antonio Casado Herraez, Benedict Benigno, Holly Guy, Mansoor R. Mirza

Provision of study materials or patients: Ursula A. Matulonis, Trine J. Nøttrup, Paul Bessette, Sven Mahner, Marta Gil-Martin, Robert M. Wenham, Susie Lau, Frederik Marmé, Antonio Casado Herraez, AnneClaire Hardy-Bessard, Joseph Buscema

Collection and assembly of data: Ursula A. Matulonis, Lydia Walder, Trine J. Nøttrup, Paul Bessette, Sven Mahner, Marta Gil-Martin, Elsa

Kalbacher, Kathrine Woie, Susie Lau, Frederik Marmé, Antonio Casado 
Herraez, Anne-Claire Hardy-Bessard, Gabriel Lindahl, Joseph Buscema, Karin Travers, Holly Guy, Mansoor R. Mirza

Data analysis and interpretation: Ursula A. Matulonis, Lydia Walder, Sven Mahner, Marta Gil-Martin, Jonathan A. Ledermann, Robert M. Wenham, Frederik Marmé, Antonio Casado Herraez, Susana Banerjee, Karin Travers, Holly Guy, Mansoor R. Mirza

Manuscript writing: All authors

Final approval of manuscript: All authors

Accountable for all aspects of the work: All authors

\section{ACKNOWLEDGMENT}

The authors thank Mark Fisher for assistance in the statistical analysis and editing (FIECON, Ltd), Heather Ostendorff-Bach, PhD, TESARO: A GSK Company (Waltham, MA), for coordinated medical writing support, and Adrienne M. Schreiber, of TESARO: A GSK Company, who edited and styled the manuscript.

\section{REFERENCES}

1. International Agency for Research on Cancer: Population fact sheets. http://globocan.iarc.fr/Pages/fact_sheets_population.aspx

2. National Cancer Institute: Cancer stat facts: Ovarian cancer. https://seer.cancer.gov/statfacts/html/ovary.html

3. Ovarian Cancer Research Alliance: Recurrence. https://ocrfa.org/patients/about-ovarian-cancer/recurrence/

4. Wright AA, Bohlke K, Armstrong DK, et al: Neoadjuvant chemotherapy for newly diagnosed, advanced ovarian cancer: Society of Gynecologic Oncology and American Society of Clinical Oncology clinical practice guideline. J Clin Oncol 34:3460-3473, 2016

5. US Food and Drug Administration: Niraparib (ZEJULA). https://www.fda.gov/drugs/informationondrugs/approveddrugs/ucm548487.htm

6. European Medicines Agency: Niraparib. Annex 1: Summary of product characteristics. http://www.ema.europa.eu/docs/en_GB/document_library/EPAR__Product_Information/human/004249/WC500239289.pdf

7. Mirza MR, Monk BJ, Herrstedt J, et al: Niraparib maintenance therapy in platinum-sensitive, recurrent ovarian cancer. N Engl J Med 375:2154-2164, 2016

8. Oza AM, Matulonis UA, Malander S, et al: Quality of life in patients with recurrent ovarian cancer treated with niraparib versus placebo (ENGOT-OV16/NOVA): Results from a double-blind, phase 3, randomised controlled trial. Lancet Oncol 19:1117-1125, 2018

9. Gelber RD, Goldhirsch A: A new endpoint for the assessment of adjuvant therapy in postmenopausal women with operable breast cancer. J Clin Oncol 4: 1772-1779, 1986

10. Friedlander M, Gebski V, Gibbs E, et al: Health-related quality of life and patient-centred outcomes with olaparib maintenance after chemotherapy in patients with platinum-sensitive, relapsed ovarian cancer and a BRCA1/2 mutation (SOLO2/ENGOT Ov-21): A placebo-controlled, phase 3 randomised trial. Lancet Oncol 19:1126-1134, 2018

11. RStudio: Open source and enterprise-ready professional software for R. https://www.rstudio.com/

12. National Institute for Health and Care Excellence: Single technology appraisal: Niraparib for maintenance treatment of relapsed, platinum-sensitive ovarian, fallopian tube and peritoneal cancer. https://www.nice.org.uk/guidance/ta528/documents/committee-papers-3

13. National Institute for Health and Care Excellence: NICE Decision Support Unit: Technical support document 14. http://nicedsu.org.uk/wp-content/uploads/ 2016/03/NICE-DSU-TSD-Survival-analysis.updated-March-2013.v2.pdf

14. Gourley C, Friedlander M, Matulonis UA, et al: Clinically significant long-term maintenance treatment with olaparib in patients (pts) with platinum-sensitive relapsed serous ovarian cancer (PSR SOC). J Clin Oncol 35, 2017 (suppl; abstr 5533) http://meetinglibrary.asco.org/record/153389/abstract

15. Royston P, Parmar MK: Flexible parametric proportional-hazards and proportional-odds models for censored survival data, with application to prognostic modelling and estimation of treatment effects. Stat Med 21:2175-2197, 2002

16. Colombo N, Lorusso D, Scollo P: Impact of recurrence of ovarian cancer on quality of life and outlook for the future. Int J Gynecol Cancer 27:1134-1140, 2017

17. Hanker LC, Loibl S, Burchardi N, et al: The impact of second to sixth line therapy on survival of relapsed ovarian cancer after primary taxane/platinum-based therapy. Ann Oncol 23:2605-2612, 2012

18. National Institute of Health and Care Excellence: Niraparib for maintenance treatment of relapsed, platinum-sensitive ovarian, fallopian tube and peritoneal cancer. https://www.nice.org.uk/guidance/ta528

19. Healthcare Improvement Scotland; Scottish Medicines Consortium: Niraparib tosylate monohydrate (Zejula). https://www.scottishmedicines.org.uk/medicinesadvice/niraparib-tosylate-monohydrate-zejula-fullsubmission-134118/

20. Berek JS, Matulonis UA, Peen U, et al: Safety and dose modification for patients receiving niraparib. Ann Oncol 29:1784-1792, 2018 
Niraparib Maintenance Treatment Improves Time Without Symptoms or Toxicity (TWiST) Versus Routine Surveillance in Recurrent Ovarian Cancer: A TWiST Analysis of the ENGOT-OV16/NOVA Trial

The following represents disclosure information provided by authors of this manuscript. All relationships are considered compensated. Relationships are self-held unless noted. I = Immediate Family Member, Inst = My Institution. Relationships may not relate to the subject matter of this manuscript. For more information about ASCO's conflict of interest policy, please refer to www.asco.org/rwc or ascopubs.org/jco/site/ifc.

Ursula A. Matulonis

Consulting or Advisory Role: Merck, Geneos, 2X Oncology, Myriad Genetics, Fujifilm, Mersana, Immunogen

Research Funding: Merck, Novartis, TESARO: A GSK Company, Syndax

Lydia Walder

Consulting or Advisory Role: FIECON, Ltd

Trine J. Nøttrup

Travel, Accommodations, Expenses: Roche

Paul Bessette

Research Funding: TESARO: A GSK Company, Merck, AstraZeneca, Clovis Oncology, Merck

\section{Sven Mahner}

Honoraria: Genentech, AstraZeneca, PharmaMar, Medac, Jenapharm, Janssen-Cilag, Teva, GlaxoSmithKline, Clovis Oncology, Novartis, Pfizer Consulting or Advisory Role: Roche, AstraZeneca, Merck Sharp \& Dohme, Janssen-Cilag, TESARO: A GSK Company, Medac, Sensor Kinesis, Clovis Oncology, Immunogen

Research Funding: Roche, AstraZeneca, Boehringer Ingelheim, GlaxoSmithKline, Janssen-Cilag, Medac, PharmaMar, TESARO: A GSK Company, Bayer

Elsa Kalbacher

Travel, Accommodations, Expenses: AstraZeneca, TESARO: A GSK Company, Sanofi, GlaxoSmithKline, Novartis, LEO Pharma

\section{Jonathan A. Ledermann}

Honoraria: Roche, AstraZeneca, Medlmmune

Consulting or Advisory Role: AstraZeneca, Medlmmune, Clovis Oncology, Pfizer, Cristal Therapeutics, Artios, Seattle Genetics, TESARO: A GSK Company, Merck

Speakers' Bureau: Clovis Oncology, Pfizer

Research Funding: AstraZeneca (Inst), MSD Oncology (Inst), TESARO: A GSK Company

Other Relationship: Regeneron

\section{Robert M. Wenham}

Stock and Other Ownership Interests: Ovation Diagnostics

Honoraria: TESARO: A GSK Company

Consulting or Advisory Role: Mersana, Merck, TESARO: A GSK Company, Clovis Oncology, Genentech

Speakers' Bureau: TESARO: A GSK Company, Clovis Oncology, Genentech

Research Funding: Merck (Inst), Prescient Therapeutics (Inst)

Travel, Accommodations, Expenses: Taplmmune

Other Relationship: AstraZeneca

\section{Frederik Marmé}

Honoraria: Genentech, Novartis, Pfizer, AstraZeneca, TESARO: A GSK Company, Clovis Oncology, Eisai, Celgene, Genomic Health, PharmaMar, Amgen, CureVac, MSD Oncology

Consulting or Advisory Role: AstraZeneca (Inst), TESARO: A GSK Company, Pfizer, Roche (Inst), Novartis, Genomic Health, CureVac, Amgen, Vaccibody (Inst), Immunomedics (Inst), Celgene, Eisai, Janssen-Cilag

Research Funding: Genentech (Inst), Novartis (Inst), AstraZeneca (Inst), Eisa (Inst), TESARO: A GSK Company (Inst), Clovis Oncology (Inst), MSD Oncology (Inst), Vaccibody (Inst)

Travel, Accommodations, Expenses: Roche, Pfizer, Novartis, PharmaMar, AstraZeneca

\section{Antonio Casado Herraez}

Consulting or Advisory Role: Roche Spain, PharmaMar

Travel, Accommodations, Expenses: PharmaMar, Roche

\section{Anne-Claire Hardy-Bessard}

Consulting or Advisory Role: Roche, AstraZeneca, TESARO: A GSK Company, Pfizer, Novartis

Travel, Accommodations, Expenses: Roche, TESARO: A GSK Company

\section{Susana Banerjee}

Honoraria: Roche

Consulting or Advisory Role: AstraZeneca (Inst), Roche (Inst), GamaMabs

Pharma, AstraZeneca, Medlmmune, TESARO: A GSK Company, TESARO: A GSK Company (Inst), Clovis Oncology (Inst), Clovis Oncology, Merck, Seattle Genetics

Research Funding: AstraZeneca (Inst), Janssen-Cilag (Inst)

Travel, Accommodations, Expenses: AstraZeneca, Clovis Oncology, TESARO: A GSK Company

\section{Benedict Benigno}

Speakers' Bureau: AstraZeneca, TESARO: A GSK Company, Clovis Oncology, Merck

Expert Testimony: Taurig Greenberg

\section{Joseph Buscema}

Employment: Arizona Oncology Associates

Leadership: Arizona Oncology Associates

Stock and Other Ownership Interests: McKesson

Honoraria: Caris Life Sciences

Consulting or Advisory Role: Caris Life Sciences

Karin Travers

Employment: TESARO: A GSK Company

Holly Guy

Consulting or Advisory Role: FIECON, Ltd

Mansoor R. Mirza

Leadership: Karyopharm Therapeutics, Sera Prognostics

Stock and Other Ownership Interests: Karyopharm Therapeutics, Sera Prognostics

Honoraria: Roche, Advaxis, AstraZeneca, Cerulean Pharma, Clovis Oncology, Novocure, Pfizer, TESARO: A GSK Company

Consulting or Advisory Role: AstraZeneca, Cerulean Pharma, Clovis Oncology, Genmab, Karyopharm Therapeutics, Novocure, Pfizer, TESARO: A GSK Company, BioCad, Sotio

Research Funding: AstraZeneca (Inst), Boehringer Ingelheim (Inst), Pfizer (Inst), TESARO: A GSK Company (Inst), Clovis Oncology (Inst)

Travel, Accommodations, Expenses: AstraZeneca, Karyopharm Therapeutics, Pfizer, Roche, TESARO: A GSK Company, SeraCare

No other potential conflicts of interest were reported. 